Price, S. (2017) Digital Museum Installations: The Role of the Body in Creativity. In (eds) S. Broadhurst \& S. Price Digital Bodies: Creativity and Technology in the Arts and Humanities, Palgrave MacMillan

\title{
14. Digital museum installations: The role of the body in creativity
}

\author{
Sara Price
}

\begin{abstract}
Digital technologies offer opportunities for creating new experiences that enable interaction that is grounded in physical action, manipulation and physical location through sensors and context aware capabilities. This brings new ways of communicating and engaging with the world that can remediate meaning making through embodied forms of interaction. This chapter draws on multimodality to explore young children's physical engagement with a digital museum art installation, illustrating how children use their bodies creatively in interaction with the exhibit, communication with one another, expression of ideas through action, and construction of a joint narrative.
\end{abstract}

\section{Introduction}

Museums increasingly employ digital technology to develop new interactive spaces to enhance visitor engagement. Sensor and context aware technologies, such as Kinect, bring new design opportunities that foster bodily interaction and supports the remediation of meaning making through embodied forms of interaction. Whole body interaction (WBI) spaces such as this incorporate key features that are thought to 
foster creative engagement, illustrating their potential to be 'creative spaces'. While digital technologies provide opportunities for new ways of being creative (Loveless, 2002), through blogs, game design, and video, audio and collaborative tools, we know little about the role of WBI experiences in promoting creativity through embodied interaction.

Within the landscape of digital technology, Dourish (2001) proposes embodied interaction is "the creation, manipulation and sharing of meaning through engaged interaction with artefacts" (p. 126). This idea of embodiment highlights the coconstruction of meaning (socially negotiated) through engaging (bodily) with the technology (artefact). This chapter draws on multimodality (which examines meaning making that is socially, physically and discursively constructed through interaction with the world) to examine two children's interaction and narrative development during their engagement with a purpose built whole body installation designed to increase visitors' awareness of Chinese Art at the London Victoria and Albert Museum. Specifically it examines how the digital and communicational resources are used, with a focus on the role of bodily engagement and interaction in the meaning making process, and how this contributes to creative narrative construction. Since Loveless (2007) cautions against the notion that free play with digital technologies will automatically foster creativity, the analysis identifies the forms of bodily interaction this design supports, specifically examining incidences across the data when the children used the space and resources to create their own narrative story. Through this, the chapter aims to illustrate ways in which a WBI environment can engender creativity, through the co-construction of shared meaning that is mediated by bodily interaction in a museum installation. 


\section{Creativity}

Fostering creativity is considered fundamentally important in developing key skills in our rapidly changing world (Burn, 2010). While perspectives on creativity differ (Begettho and Kaufmann, 2009), this chapter, being concerned with young children's creativity, draws on notions of everyday creativity: little- $c$ and mini-c constructs. Little-c theories emphasise creative expression, through inquisitiveness, imagination and freedom, where a "group or individual produces a perceptible product that is both novel and useful as defined within a social context" (Plucker et al., 2004, p.90). Minic emphasises creative interpretation and ideation, including any new, personally meaningful interpretation of an experience (Begetto and Kaufmann 2007). This category enables the attribution of creativity to younger people who may not have the skills or knowledge of more expert people, yet still make creative steps in meaning making through interpretation.

Mini and little-c constructs align with theories of play and creativity, and Vygotskian ideas of cognitive and creative development, thus addressing criticisms of these constructs that highlight the nonsensical notion that all everyday experiences can be counted as creative (Banaji et al., 2010). According to Vygotsky imagination is an essential foundation for creativity and is dependent on the breadth and depth of an individual's experience since "imagination always builds using materials supplied by reality" (Vygotsky (1930/2004, p. 14, cited Tsai, 2012). Imagination is embedded in pretend play, specifically through the use of symbolic tools in making meaning. Symbolic play is linked to abilities of divergent and associative thinking (e.g. Russ, 
2003), and abstract thinking, which also form the basis for childhood creativity (Sefer, 2013), and the development of adult creativity, since both are embedded in imaginary thought and supposition (Carruthers, 2002).

Collective literature distinguishes various qualities linked to increased creative potential: openness to new experiences, willingness to be surprised, active observation, exploring the unknown (Kaufmann and Begetto, 2009); experience and repetition (Slutzkaya, 2006), curiosity and exploration, and enabling children to make decisions (Harrington and Block, 1987). However, there is little empirical work on embodied creativity, Stanciu (2015) highlighting this as an under researched area. According to Kokas (1999, cited Vass 2011) children's spontaneous movement inspired by music can lead to collective images, stories and narratives. More recently, Slepian and Ambady claim that bodily movement "can influence cognitive processing, with fluid movement leading to fluid thinking" (21012:628) or creativity. Vass (2011) also notes non-language based dimensions of intersubjectivity and collective imagination in the context of music creation. Here she highlights ideas of shared creative engagement evidenced through 'spontaneously coordinated shared movement', 'shared chain of association', and mutual influence on action.

Although, research in digital WBI experiences shows how bodily based forms of interaction increase engagement and immersion (Adachi et al., 2013; Zuckerman and Gal-Oz, 2013) and support social interaction and learning (Jakobsen and HornbÆk, 2014; Antle et al., 2011), there has been little focus on the ways in which children use the designed digital resources to support creative expression and interpretation. 


\section{The installation}

Digital Dragons, created by Bright Ideas Design in collaboration with the museum, was based on two Chinese paintings: 'Nine Dragons' by Chen Rong and 'Farewell to Xunyang' by Qiu Ying. The installation used four projectors, Xbox Kinect and custom code to create an interactive projection derived from each painting, placed in an empty room with a wide entrance space along one wall. A wall projection was distributed around the other three walls, and a floor projection sat within the wallprojected area. The V\&A installation brief was to: encourage interaction between people within the space; engage visitors in the content and context of classical Chinese painting; be playful, contemporary and exciting, providing routes into learning other than through absorption of knowledge in labels; provide a memorable, immersive experience; challenge preconceived ideas of how people interact with collections in museums, discouraging passiveness and encouraging activity and interaction with artworks and with other people.

$<$ FIGURE 14.1 ABOUT HERE> Caption to read: Figure 14.1 Interaction in the Digital Dragons installation based on Chen Rong's 'Nine Dragons' painting, MODE, 2014

The installation design offered new experiences in relation to pieces of art, through physical action that mediated dynamic changes to visualisations based on the paintings. The experience inspired by the Nine Dragons painting comprised a floor projection with constant twirling spots, like whirlwinds, and transient coloured pearls (or spots) that, when stood on, elicited an animation of pearls that moved across the 
wall display each being chased by a dragon (figure 14:1). The experience inspired by the Farewell to Xunyang painting comprised a floor projection with moving flowers, blue areas (like water) and transient symbols that appeared on the floor (e.g. boat, horse, moon, hands) (figure 14:2). Standing on these symbols elicited animated changes to the painting, for example, the boat moved, the horse neighed, people waved).

$<$ FIGURE 14.2 ABOUT HERE> Caption to read: Figuer 14.2 Interaction in the Digital Dragons installation with the digital depiction of Qui Ying's painting 'Farewell to Xunyang', MODE 2014

Price et al., (2015) showed that this installation fostered features of interaction that map to mini-c constructs such as, active observation, exploration of the unknown, freedom of movement (Slepain and Ambady, 2012), opportunity for repetition (Slutzkaya, 2006), encouraging curiosity (Harrington and Block, 1987) and shared or collaborative forms of action and interaction (Vass, 2011). This chapter moves beyond informing design (Price et al., 2015) to examine the creative process engendered by bodily interaction.

\section{Analytical approach}

Researching creativity commonly relies on evaluating creative products (NAACE report) or verbal articulation to assess children's creative ideas (e.g. Vass et al., 2014), with little focus on the role of the body in creative communication. While some studies have used patterns of gaze (D'Mello et al., 2012), hand movements (Antle et 
al., 2009), and unfolding bodily behaviours, such as gaze, gesture and movement (Price et al, 2015) to understand how interaction is shaped, WBI research typically uses retrospective measures of engagement (Pares and Malinvieri, 2014), rather than observing individuals interacting. In contrast, multimodality attends to the different ways of making meaning with the various 'texts' or semiotic resources that are made available to them (Kress, 1997). Derived from social semiotic theory, "semiotic resources are the actions, materials and artifacts we use for communicative purposes" (van Leeuwen 2004:285). These include bodily posture and movement, facial expressions and gestures, writing, images. In the context of this chapter, these combine in a multimodal ensemble of computer graphics dynamically linked to action through sensor technologies. It "emphasizes situated action...with attention to people's situated choice of resources." (Jewitt, 2013).

With changes in technology and media, multimodal practices have shifted from a culture dominated by word-based forms of communication to a culture infused with visual forms. Digital technologies have brought new emphasis to bodily modes of communication through gestural and touch interfaces, changing the potential for communication and interaction to take place through bodily action.

Here we use a multimodal analytical approach to examine Alice and Bryony (aged five years) interacting with the Digital Dragons installation. The analysis focuses on how the resources to shape their bodily interaction and how they are remediated and incorporated into their collective narrative, to explore the role of the body in creative forms of interpretation and expression. This involved iterative viewing of video data of two key episodes of interaction. One episode comprised Alice interacting with the 
Dragons followed by the Landscape and then the Dragons once more. The second episode comprised Alice, with her friend Bryony, interacting with one iteration of the Landscape experience.

A written transcript detailed the sequences of action related to each resource, the space as a whole and any talk. The transcript was used with the video to identify the kinds of movements in relation to different resources, trajectories of action, and linking to the constructed narrative in the second episode, where a joint narrative was built around the pair's interaction.

\section{Interaction description}

Our findings begin with episode one describing how Alice physically engaged with the resources, where no verbal expression occurred. This is followed by a description of the joint narrative that Alice and Bryony created through their actions and verbal communication, identifying how the resources were repurposed in episode two.

\section{Episode 1 description:}

The landscape experience begins as Alice walks into the interactive space. Traditional Chinese music is playing. Alice looks at the floor, then the wall. She walks towards the wall, watching the painting and wanders along it swinging her arms. She turns to the look at the floor, where a coloured spot appears; runs and jumps onto the spot, then turns to look at the wall again. She walks over to the horse on the wall projection, lifts her hand as if to stroke it, walks to the image of a boat with people, then returns to the floor projection. She stands on a 'boat image' spot on the floor, and turns to 
look at the wall, while making a ballet movement with her arms. She then engages in a repeated pattern of standing on floor symbols and looking at the wall projection: as she stands on the horse symbol the horse on the wall neighs, the sound drawing her attention to wall. During this period she seeks out floor symbols as they appear (horse, tree, boat, moon, hands) and stands on them. When she stands on the hands symbol she looks to the wall and watches the people on the projection wave. She walks forward to the boat on the wall and waves back to the people on the boat. She then uses her body differently, touching the blue area on the floor with her foot; she kneels down by the hands symbol and places her hands crossed as the image shows; she holds her hands up and moves them up and down, then around over the symbol image (like a magician conjuring).

As the projection changes Alice makes small hand and dance-like body movements. She watches the dragon appear on the wall projection, runs towards it and touches it. She places her hands in a prayer position and swings her body from side to side. She notices a yellow spot appear on the floor and walks towards it, but as a dragon roars and flies across the wall she turns and moves towards that. She then touches the dragon and follows it across the wall, running to keep up with it moving. When it disappears she goes back to the other side of the room, to do the same with the next dragon. Here the animation guides her bodily movement and trajectory. As if having familiarised herself with the wall projection, she then moves her attention to the floor, since she knows from the landscape interaction that there are links between interaction with the floor and the wall projection. She focuses on the floor, as if trying to get familiar with the floor symbols and changes. The coloured spots (appearing and disappearing) elicit movement from one place to another, exploration through 
touching, standing and stepping. On the twirling grey areas (constant) she twists her body in a half twirl with arms out, then a full twirl. She then alternates her attention between floor and wall, adopting specific bodily postures: raising her arms out to the side then bringing them into a praying stance, and crossing her hands over her chest, perhaps inspired by the music.

The projection then moves back to the landscape. Alice looks excited, happily surprised. During this second iteration she spends more time looking at the flowers and blue area on the floor projection, kneeling at the edge of the 'water' touching this space. She engages with the boat people in the wall projection again, bringing in bodily movements she expressed during the dragon display i.e praying stances, but this time combining them with a kneeling posture, and bowing with hands placed in prayer position. Towards the end she makes several twirls across the interactive space towards the painting ending in a kung-fu like pose. Here the context of the wall projection, the animated characters and the music appeared to inspire culturally related forms of bodily expression.

\section{Episode 2 description}

The Landscape experience is running. Bryony notices the paintbrush symbol on the floor, taps it with her foot and calls to Alice. Alice says "we must take this information to the village". The notion of the village potentially taken from the boat and people in the wall projection provides the context for their imaginary narrative. They note there are many flowers on floor, and attribute this to signal 'danger'. Bryony then uses a character from the wall projection: "look, that man is riding to collect more water for his family". Alice elaborates on this "that man is riding to get 
water from the dangerous caverns. Dangerous caverns are where the dragons sleep. We have to warn them". Alice thus builds on Bryony's idea, at the same time bringing in concepts from her previous interaction with the Dragon experience.

They then engage in a form of ritual: lift up their arms and 'welcome the spirit', place their hands in prayer, followed by spitting on their hands and rubbing together in a washing mime. Alice: "we have to rinse our hands, its exactly like soap and we rinse our hands clean, just once or twice". They then focus on trying to get water for the villagers, who cannot get it because of the dragon caves. Alice has previously engaged with the blue areas on the floor projection, touching them with her foot and hands, bringing ideas of water. "We need to get some water from the, from the, from the sky"; and "lets run and run and look for the taps". They run around the edge of the interactive floor space, stop with Alice saying "tschhh" like the sound of water, and miming turning on a tap, and "get some from the lake: beautiful and sparkling", referring to the blue on the floor projection. After doing this for a while Bryony stumbles (as if carrying something heavy) towards the horse and man on the wall projection, saying "here you are". Alice moves along the edge of the wall projection making 'tschhh' noises as if squirting something onto the painting, and says, "we put water all over the village. Lots more dragons, lots more danger”.

Then the hands symbol appears on the floor and Bryony says this means 'no more', while crossing her hands like the symbol on the floor. Alice draws on her previous interaction saying "when the hand comes, you wave", and makes a wave gesture. The projection finishes. 


\section{Creative development through the body}

Throughout episode one the resources engendered creative interaction. Initially the images on both the floor and wall drew Alice's attention and generated active observation and exploring the unknown (Kaufmann \& Begetto, 2009) through her bodily actions and movement, e.g. following the trajectory of the dragon; touching and interacting directly with characters on the landscape, and through repeated action trajectories (Slutzkaya, 2006). The people's dress, landscape, music, and use of dragons all map to notions of what it is to be Chinese, and the freedom of movement (Slepain and Ambady, 2012) that the interaction allows enabled Alice to explore through her body posture and movements in ways that introduced culturally related ideas in her bowing and praying stances, twirling and kung-fu poses.

In the second episode bodily exploration and experience featured in the constructed narrative, evidenced both through actions and talk. Vygotsky (1967) emphasises the use of tools in making meaning and symbolic play. The semiotic resources made available through the installation, and those that the children themselves bring to their interaction with it, are tools that the children used in their meaning making. Kress (2010) emphasizes how resources are constantly transformed, combined and shaped by humans. Here we can see how the resources selected formed the foundation for the children's imagined narrative. Several aspects of the landscape were brought into the story: the notion of a village (derived from group of people on the boat); people's imagined activity of needing water, but not able to get it (horse and man, who they claim have 'stopped'); and areas on the image that looked like caves helped bring the two experiences together, with the caves as imagined 'dens' for dragons. These 
resources were supplemented with imaginary artefacts like the bucket for collecting water, and abstract notions of 'danger' linked to dragons. Meaning is thus transformed through the re-use of characters in both experiences: dragons from one experience linked to the people, horse and boat from the other to create a new interpretation and narrative. The attention to and role of cultural ideas also emerges. This is initially apparent in the Alice's bodily expression of culturally influenced ideas through bodily postures of bowing with her hands together, and Kung-fu poses. Later their narrative sits in the context of Chinese culture, but also draws on cultural notions of Western fairy tales involving dragons and ideas of danger, with people in need of help, and the girls coming to their rescue. Yet this Western notion of the dragon as symbolizing danger sits in contrast to Chinese dragons, which are considered benevolent, and signify power, strength and good luck. This highlights the influence of cultural experience on interpretation, and the cultural shaping of meaning, but also the potential for this form of interaction to better foreground other cultural beliefs about characters, like dragons, exposing visitors to alternative cultural ideas, thus extending the potential for creativity.

The analysis demonstrates the children's ability "to think about affect-laden fantasy and the capacity to experience emotion" (Russ, 2003, p. 292-293), which are important for creativity, and show how their play facilitated insight and divergent thinking (ibid). Vass' (2011) notions of creativity focus on how collaborators influence one another in creative contexts through joint chain of associations, growing story together, and collective pooling of ideas, characterised by overlaps, interruptions, and speedy exchanges. These features are evident in these episodes through bodily interaction and communication, and the unfolding collective imaginary narrative. 
Thus, in contrast to 'mere' digital creations that Loveless claims can be 'empty showiness', the example here demonstrates a 'representation of meaning' i.e., the children are making a meaningful narrative through their physical engagement with the digital resources thus 'elevating the production to a position beyond the merely decorative' (Loveless and Taylor, 2000, 65). The idea of 'product', here, due to the nature of the interaction, is however ephemeral - it is not a persistent resource, but a narrative that is transient, and therefore only tangible to the children engaging in that narrative. In terms of evaluation, then, and objective ideas of 'value' (e.g. Loveless, 2007) this creates a challenge if we think about creativity as a 'product' in the physical sense, like a painting, or music, that is re-accessible by others. In contrast, this study focuses on notions of 'internal' creativity, and therefore speaks more to theories of 'foundational creativity' or processes that are considered important in developing 'creative expertise'. Indeed, Banaji's (2010) review suggests that if we want to facilitate imaginary thought and supposition that form the roots of adult creativity (Carruthers, 2002) enabling playful exploration experiences with ideas, artefacts and materials is important. It could be argued, then that this museum installation provides such a space through the physical interaction with digital artefacts and materials. In particular the analysis shows how the installation cultivated 'play as imagination in action', and thus creative through a real situation taking 'new and unfamiliar meaning' (Vygotsky, 1995, cited Lindqvist, 2003).

\section{Conclusion}


This chapter illustrates how bodily engagement with digital-physical resources can lead to creative expression and interpretation through the development of new narrative mediated by the interaction. The digital resources supported creative expression: mimicking (watching other's actions and copying), repetition (patterns of interaction), exploration ( of links between action and wall projection, ideas), and use of different bodily movements (e.g. jumping, running, waving, touching). This analysis also shows how WBI provides resources that foster symbolic play and joint narrative development and how bodily action and engagement can lead to creative expression, interpretation and composition. It demonstrates how children 'create, manipulate and share meaning' through engaging bodily within a digital-physical space, and how 'embodied interaction' is central to a collaborative creative process. The idea of being able to control events through bodily movement enables intentionality in communication and engagement. The physical modality enabled direct interaction with the characters on the image (gesturally and verbally), bodily expressions of Chineseness, and the potential for acting out ideas through physical movement. Collectively this fostered immersion in the experience and contributed to and shaped the narrative development through 'spontaneously coordinated shared movement' and 'shared chain of association' (Vass, 2011). Physical engagement also led to a more extended time spent with the digital resources, providing space for ideas to develop, and the potential for fostering free-flow of ideas.

\section{Acknowledgements}


This research was supported by Economic and Social Research Council (RES-576-250027). Thanks also go to the Victoria and Albert Museum, London and the children and families who participated in the research.

\section{References}

Banaji, Shakuntala, Andrew and Buckingham, David. (2010) 'The Rhetorics of Creativity: A literature review, $2^{\mathrm{m}}$ Edition' Creativity Culture and Education Series. Arts Council UK.

Carruthers, Peter (2002) 'Human Creativity: its cognitive basis, its evolution and its connection with child pretence' British Journal for the Philosophy of Science, 53(2) $225-249$

Dourish, Paul (2001) Where the action is: Foundations of embodied interaction, Cambridge, MA: MIT Press.

Harrington, David, Block, Jeanne and Block, Jack (1987) 'Testing aspects of Carl Rogers's theory of creative environments: Child-rearing antecedents of creative potential in young adolescents' Journal of Personality and Social Psychology, 52, $851-856$.

Jewitt, Carey (2013) 'Multimodal methods for researching digital technologies' in Sara Price, Carey Jewitt, Barry Brown (Eds) The SAGE Handbook of Digital Technology Research (pp. 250-265). Los Angeles: SAGE.

Kaufmann, James and Begetto, Ronald (2009) 'Beyond big and little: the four C model of creativity' Review of General Psychology. 13 (1) 1-12 Kress, Gunther (1997) Before writing: Rethinking the paths to literacy. New York: Routledge 
Lindqvist, Gunilla (2003) 'Vygotsky's Theory of Creativity', Creativity Research Journal, 15(2): 245-251

Loveless, Avril (2007) Creativity, new technologies and learning: an update Bristol: Futurelab.

Price, Sara, Jewitt, Carey and Sakr, Mona (2015) 'Exploring Whole-body Interaction and Design for Museums' Interacting with Computers first published online September 25, 2015 doi:10.1093/iwc/iwv032

Russ, Sandra (2003) 'Play and Creativity: developmental issues' Scandinavian Journal of Educational Research 47(3) 291-303

Sefer, Jasmina (2013) ‘Symbolic Play and Analogy: A Way to Foster Children’s Creativity' Creativity and (Early) Cognitive Development: Papers from the 2013 AAAI Spring Symposium

Slepian, Michael L., \& Ambady, Nalini (2012) 'Fluid movement and creativity' Journal of Experimental Psychology: General, 141, 625-629.

Slutskaya, Natalie (2006) 'Creativity and Repetition' Creativity and Innovation Management, 15(2) 150-156.

Stanciu, Marius (2015) 'Embodied Creativity: a critical analysis of an underdeveloped subject' Procedia - Social and Behavioural Sciences, 187, 312-317 Kuan Chen Tsai (2012) Play, Imagination, and Creativity: A Brief Literature Review Journal of Education and Learning; Vol. 1, No. 2, 15-20

Van Leeuwen, Theo (2004) Introducing Social Semiotics: An Introductory Textbook. London: Routledge

Vass, Eva (2011) 'Metamorphosis: Creativity, Connectedness, embodiment and affect' International Symposium on the Language of Art and Music, Berlin, Academy for Cultural Diplomacy 
Vass, Eva, Littleton, Karen, Jones, Ann \& Miell, Dorothy (2014) 'The affectively constituted dimensions of creative interthinking' International Journal of Educational Research. 66, 63-77

Von Held, Felix (2011) Collective Creativity: Exploring creativity in Social Network development as part of organisational learning. Springer Vygotsky, Lev (1967) 'Play and Its Role in the Mental Development of the Child' Soviet Psychology, 5(3) 6-18 